\title{
Sensitivity Analysis of the Critical Speed in Railway Vehicle Dynamics
}

\author{
Bigoni, Daniele; True, Hans; Engsig-Karup, Allan Peter
}

Published in:

Proceedings of IAVSD2013

Publication date:

2013

Link back to DTU Orbit

Citation (APA):

Bigoni, D., True, H., \& Engsig-Karup, A. P. (2013). Sensitivity Analysis of the Critical Speed in Railway Vehicle Dynamics. In S. Iwnicki (Ed.), Proceedings of IAVSD2013 [28.2]

\section{General rights}

Copyright and moral rights for the publications made accessible in the public portal are retained by the authors and/or other copyright owners and it is a condition of accessing publications that users recognise and abide by the legal requirements associated with these rights.

- Users may download and print one copy of any publication from the public portal for the purpose of private study or research.

- You may not further distribute the material or use it for any profit-making activity or commercial gain

- You may freely distribute the URL identifying the publication in the public portal

If you believe that this document breaches copyright please contact us providing details, and we will remove access to the work immediately and investigate your claim 


\title{
SENSITIVITY ANALYSIS OF THE CRITICAL SPEED IN RAILWAY VEHICLE DYNAMICS
}

\author{
Daniele Bigoni, Hans True, Allan P. Engsig-Karup \\ Department of Applied Mathematics and Computer Science, Technical University of Denmark \\ Matematiktorvet, building 303B \\ DK-2800 Kgs. Lyngby, Denmark \\ E-mail: dabi@dtu.dk
}

\begin{abstract}
We present an approach to global sensitivity analysis aiming at the reduction of its computational cost without compromising the results. The method is based on sampling methods, cubature rules, High-Dimensional Model Representation and Total Sensitivity Indices. The approach has a general applicability in many engineering fields and does not require the knowledge of the particular solver of the dynamical system. This analysis can be used as part of the virtual homologation procedure and to help engineers during the design phase of complex systems.

The method is applied to a half car with a two-axle Cooperrider bogie, in order to study the sensitivity of the critical speed with respect to suspension parameters. The importance of a certain suspension component is expressed by the variance in critical speed that is ascribable to it. This proves to be useful in the identification of parameters for which the exactness of their values is critically important.
\end{abstract}

\section{INTRODUCTION}

The past couple of decades have seen the advent of computer simulations for the study of deterministic dynamical systems arising from any field of engineering. The reasons behind this trend are both the enhanced design capabilities during production and the possibility of understanding dangerous phenomena. However, deterministic dynamical systems fall short in the task of giving a complete picture of reality: several sources of uncertainty can be present when the system is designed and thus obtained results refer to single realizations, that in a probabilistic sense have measure zero, i.e. they never happen in reality. The usefulness of these simulations is however proved by the achievements in Computer-Aided Design (CAD).

The studies of stochastic dynamical systems allow for a wider analysis of phenomena: deterministic systems can be extended with prior knowledge on uncertainties with which the systems are described. This enables an enhanced analysis and can be used for risk assessment subject to such uncertainties and is useful for decision making in the design phase.

In the railway industry, stochastic dynamical systems are being considered in order to include their analysis as a part of the virtual homologation procedure [1], by means of the framework for global parametric uncertainty analysis proposed by the OpenTURNS consortium. This framework splits the uncertainty analysis task in four steps:

A. Deterministic modeling and identification of Quantities of Interest (QoI) and source of uncertainties

B. Quantification of uncertainty sources by means of probability distributions

C. Uncertainty propagation through the system

D. Sensitivity analysis

Railway vehicle dynamics can include a wide range of uncertainty sources. Suspension characteristics are only known within a certain tolerance when they exit the manufacturing factory and are subject to wear over time that can be described stochastically. Other quantities that are subject to uncertainties are the mass and inertia of the bodies, e.g. we don't know exactly how the wagon will be loaded, the wheel and track geometries, that are subject to wear over time, and also external loadings like wind gusts.

In this work the QoI will be the critical speed of a fixed half-wagon with respect to uncertain suspension components (step A). The deterministic and stochastic models will be presented in section 2. Step B requires measurements of the input uncertainty that are not available to the authors, so the probability distribution of the suspension components will be assumed to be Gaussian, without losing the generality of application of the methods used in C and D.

Techniques for Uncertainty Quantification (UQ) will be presented in section 3.1. They have already been applied in [2] and [3] to perform an analysis of Uncertainty propagation (step C). They will turn useful also in section 3.2 and 3.3 for the sensitivity analysis technique to be presented (step D). This is based on Total Sensitivity Indices (TSI) obtained from the ANOVA expansion of the function associated to the QoI [4]. Section 4 will contain the results of such analysis. 

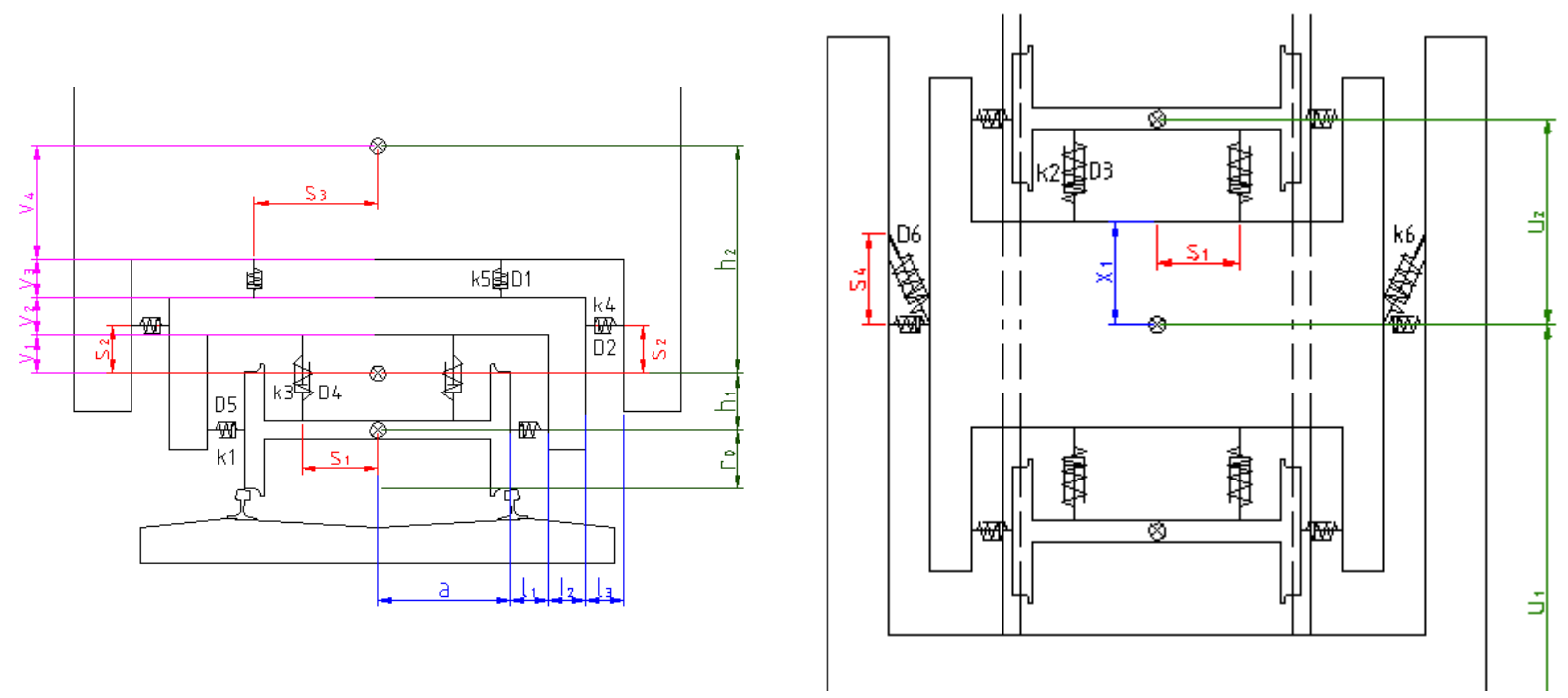

Fig. 1 The half-wagon equipped with the Cooperrider bogie.

\section{THE VEHICLE MODEL}

In this work we will consider a fixed half wagon equipped with a Cooperrider bogie, running on tangent track with wheel profile S1002 and rail UIC60. The position of the suspension components is shown in Fig. 1. In [5] a framework for the simulation of the dynamics of complete wagons running on straight and curved tracks has been implemented and tested based on the Newton-Euler formulation of the dynamical system:

$$
\begin{gathered}
\sum_{i=1}^{n} \vec{F}_{i}=m \vec{a}, \\
\sum_{i=1}^{m} M_{i}=\frac{d}{d t}([J] \cdot \vec{\omega})+\vec{\omega} \times([J] \cdot \vec{\omega}),
\end{gathered}
$$

where $F_{i}$ and $M_{i}$ are respectively the forces and the torques acting on the bodies, $m$ and $[J]$ are the mass and inertia of the bodies, $\vec{a}$ and $\dot{\vec{\omega}}$ are the acceleration and the angular acceleration of the bodies.

In this work the wagon will be fixed in order to alleviate the lateral oscillations during the hunting motion that would, in some cases, break the computations. The mathematical analysis and the generality of the methods proposed are not weakened by this assumption, even if the results may change for different settings. The wheel-rail interaction is modeled using tabulated values generated with the routine RSGEO [6] for the static penetration at the contact points. These values are then updated using Kalker's work [7] for the additional penetrations. The creep forces are approximated using Shen-Hedrick-Elkins nonlinear theory [8]. The complete deterministic system

$$
\frac{d}{d t} \mathbf{u}(t)=\mathbf{f}(\mathbf{u}, t),
$$

is nonlinear, non-smooth, and it has 28 degrees of freedom.

\subsection{Nonlinear dynamics of the deterministic model}

The deterministic dynamics of the complete wagon with a couple of Cooperrider bogies were analyzed in [5]. The stability of the half-wagon model considered in this work is characterized by a subcritical Hopf-bifurcation at $v_{L}=114 \mathrm{~m} / \mathrm{s}$, as it is shown in Fig. 2a, and a critical speed $v_{N L}=50.47 \mathrm{~m} / \mathrm{s}$. The critical speed is found using a continuation method from the periodic limit cycle detected at a speed greater than the Hopf-bifurcation speed $v_{L}$. In order to save computational time, we try to detect the periodic limit cycle at speeds lower than $v_{L}$ perturbing the system as described in [9]. This is the approach that we will take during all the computations of critical speeds in the next sections. The criterion used in order to detect the value of the critical speed is based on the power of the lateral oscillations in a $1 s$ sliding window of the computed solution. Fig. $2 \mathrm{~b}$ shows how this criterion is applied. 


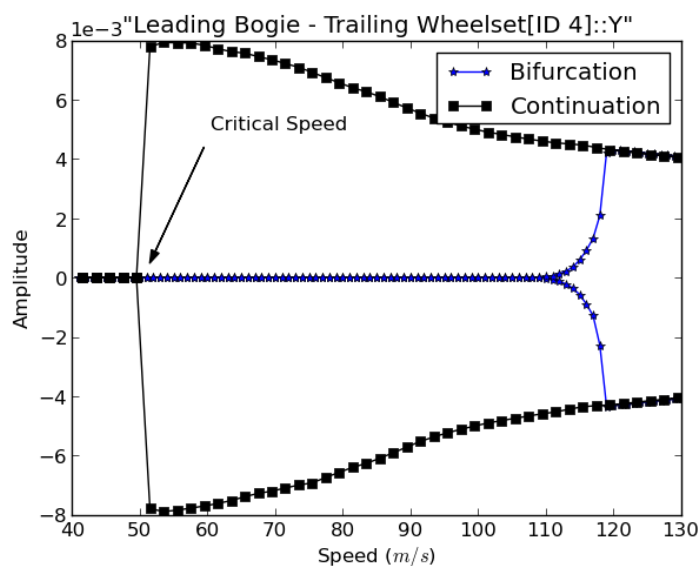

(a) bifurcation diagram

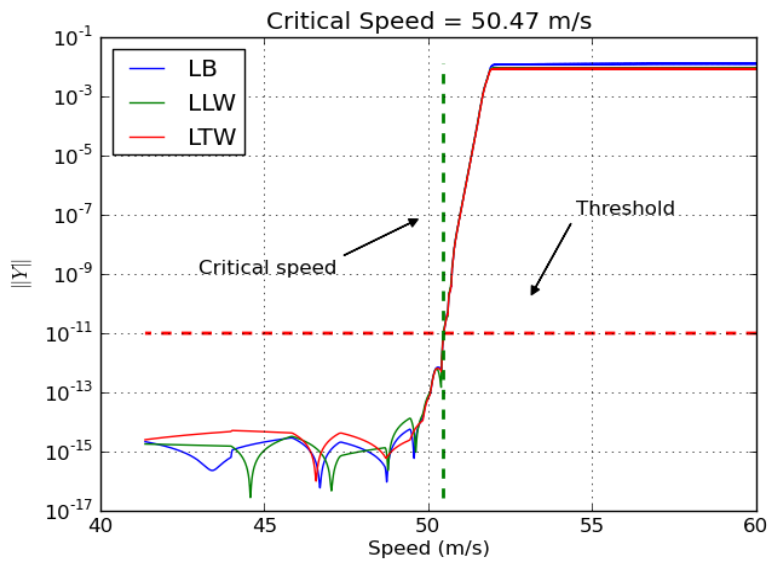

(b) Critical speed detection criteria

Fig. 2 Left: complete bifurcation diagram where the folding point is detected by continuation (ramping) method from the periodic limit cycle. Right: criterion for the determination of the critical speed based on the power of the lateral oscillations in a sliding window. LB, LLW and LTW stand for the bogie frame, the leading wheel set and the trailing wheel set respectively.

\subsection{The stochastic model}

In the following we will assume that the suspension characteristics are not deterministically known. Rather, they are described by probability distributions stemming from the manufacturing uncertainty or the wear.

If experimental information is available, then some standard distributions can be assumed and an optimization problem can be solved in order to determine the statistical parameters of such distributions (e.g. mean, variance, etc.). Alternatively the probability density function of the probability distribution can be estimated by Kernel smoothing [10].

Due to the lack of data to the authors, in this work the probability distributions associated with the suspension components will be assumed to be Gaussian around their nominal value, with a standard deviation of $5 \%$. We define $\mathbf{Z}$ to be the $d$-dimensional vector of random variables $\left\{z_{i} \sim N\left(\mu_{i}, \sigma_{i}\right\}_{i=1}^{d}\right.$ describing the distributions of the suspension components, where $d$ is called the co-dimension of the system. The stochastic dynamical system is then described by

$$
\frac{d}{d t} \mathbf{u}(t, \mathbf{Z})=\mathbf{f}(\mathbf{u}, t, \mathbf{Z}), \quad(0, T] \times \mathbf{R}^{d} .
$$

\section{SENSITIVITY ANALYSIS}

Sensitivity analysis is used to describe how the model output depends on the input parameters. Such analysis enables the user to identify the most important parameters for the model output. Sensitivity analysis can be viewed as the search for the direction in the parameter space with the fastest growing perturbation from the nominal output.

One approach of sensitivity analysis is to investigate the partial derivatives of the output function with respect to the parameters in the vicinity of the nominal output. This approach goes by the name of local sensitivity analysis, stressing the fact that it works only for small perturbations of the system.

When statistical information regarding the parameters is known, it can be embedded in the global sensitivity analysis, which is not restricted to small perturbations of the system, but can handle bigger variability in the parameter space. This is the focus of this work and will be described in the following sections.

\subsection{Uncertainty Quantification}

The solution of (3) is $\mathbf{u}(t, \mathbf{Z})$, varying in the parameter space. In uncertainty quantification we are interested in computing the density function of the solution and/or its first moments, e.g. mean and variance:

$$
\begin{gathered}
\mu_{\mathbf{u}}(t)=\mathbf{E}[\mathbf{u}(t, \mathbf{Z})]_{\rho_{\mathbf{Z}}}=\int_{\Omega^{d}} \mathbf{u}(t, \mathbf{z}) d F_{\mathbf{Z}}(\mathbf{z}), \\
\sigma_{\mathbf{u}}^{2}(t)=\mathbf{V}[\mathbf{u}(t, \mathbf{Z})]_{\rho_{\mathbf{Z}}}=\int_{\Omega^{d}}\left(\mathbf{u}(t, \mathbf{z})-\mu_{\mathbf{u}}(t)\right)^{2} d F_{\mathbf{Z}}(\mathbf{z}),
\end{gathered}
$$


where $\rho_{\mathbf{Z}}(\mathbf{z})$ and $F_{\mathbf{Z}}(\mathbf{z})$ are the probability density function (PDF) and the cumulative distribution function (CDF) respectively. Several techniques are available to approximate these high-dimensional integrals. In the following we present the two main classes of these methods.

\section{Sampling based methods}

The most known sampling method is the Monte Carlo (MC) method, which is based on the law of large numbers. Its estimates are:

$$
\begin{gathered}
\mu_{\mathbf{u}}(t) \approx \bar{\mu}_{\mathbf{u}}(t)=\frac{1}{M} \sum_{j=1}^{M} \mathbf{u}\left(t, \mathbf{Z}^{(j)}\right), \\
\sigma_{\mathbf{u}}^{2}(t) \approx \bar{\sigma}_{\mathbf{u}}^{2}(t)=\frac{1}{M-1} \sum_{j=1}^{M}\left(\mathbf{u}\left(t, \mathbf{Z}^{(j)}\right)-\bar{\mu}_{\mathbf{u}}(t)\right)^{2},
\end{gathered}
$$

where $\left\{\mathbf{Z}^{(j)}\right\}_{j=1}^{M}$ are realizations sampled randomly within the probability distribution of $\mathbf{Z}$. The MC method has a probabilistic error of $O(1 / \sqrt{M})$, thus it suffers from the work effort required to compute accurate estimates. However the MC method is very robust because this convergence rate is independent of the co-dimensionality of the problem, so it's useful to get approximate estimates of very high-dimensional integrals.

Sampling methods with improved convergence rates have been developed, such as Latin Hypercube sampling and Quasi-MC methods. However, the improved convergence rate comes at the expense of several drawbacks, e.g., the convergence of Quasi-MC methods is dependent of the co-dimensionality of the problem and Latin Hypercube cannot be used for incremental sampling.

\section{Cubature rules}

The integrals in (4) can also be computed using cubature rules. These rules are based on a polynomial approximation of the target function, i.e. the function describing the relation between parameters and QoI, so they have superlinear convergence rate on the set of smooth functions. Their applicability is however limited to low-co-dimensional problems because cubature rules based on a tensor grid suffer the curse of dimensionality, i.e. if $m$ is the number of points used in the one dimensional rule and $d$ the dimension of the integral, the number of points at which to evaluate the function grow as $O\left(m^{d}\right)$. They will however be presented here because they represent a fundamental tool for the creation of high-dimensional model representations that will be presented in the next section.

Let $\mathbf{Z}$ be a vector of independent random variables in the probability space $\left(\mathbf{D}, \mathbf{B}, F_{\mathbf{Z}}\right)$, where $\mathbf{D} \subseteq \mathbf{R}^{d}, \mathbf{B}$ is the Borel set constructed on $\mathbf{D}$ and $F_{\mathbf{Z}}$ is a probability measure (i.e. the CDF of $\mathbf{Z}$ ). For this probability measure we can construct orthogonal polynomials $\left\{\phi_{n}\left(z_{i}\right)\right\}_{n=1}^{N_{i}}$ for $i=1 \ldots d$, that form a basis for each independent dimension of $\mathbf{D}$ [11]. The tensor product of such a basis forms a basis for $\mathbf{D}$. From these orthogonal polynomials, the Gauss quadrature points and weights $\left\{\mathbf{z}_{j_{i}, \ldots, j_{d}}, \mathbf{w}_{j_{i}, \ldots, j_{d}}\right\}_{j_{i}, \ldots, j_{d}=1}^{N_{1} \ldots N_{d}}$ can be derived using the Golub-Welsch algorithm [11], obtaining approximations for (4):

$$
\begin{gathered}
\mu_{\mathbf{u}}(t) \approx \bar{\mu}_{\mathbf{u}}(t)=\sum_{j_{i}=1}^{N_{i}} \cdots \sum_{j_{d}}^{N_{d}} \mathbf{u}\left(t, \mathbf{z}_{j_{i}, \ldots, j_{d}}\right) \mathbf{w}_{j_{i}, \ldots, j_{d}}, \\
\sigma_{\mathbf{u}}^{2}(t) \approx \bar{\sigma}_{\mathbf{u}}^{2}(t)=\sum_{j_{i}=1}^{N_{i}} \cdots \sum_{j_{d}}^{N_{d}}\left(\mathbf{u}\left(t, \mathbf{z}_{j_{i}, \ldots, j_{d}}\right)-\bar{\mu}_{\mathbf{u}}(t)\right)^{2} \mathbf{w}_{j_{i}, \ldots, j_{d}} .
\end{gathered}
$$

Gauss quadrature rules of order $N$ are accurate for polynomials of order up to degree $2 N-1$. This high accuracy comes at the expense of the curse of dimensionality due to the use of tensor products in high-dimensional integration. This effect can be alleviated by the use of Sparse Grids techniques proposed by Smolyak [12] that use an incomplete version of the tensor product. However, in the following section we will see that we can often avoid working in very high-dimensional spaces.

\subsection{High-dimensional model representations}

High-dimensional models are very common in practical applications, where a number of parameters influence the dynamical behaviors of a system. These models are very difficult to handle, in particular if we consider them as black-boxes where we are only allowed to change parameters. One method to circumvent these difficulties is the 
HDMR expansion [13], where the high-dimensional function $f: \mathbf{D} \rightarrow \mathbf{R}, \mathbf{D} \subseteq \mathbf{R}^{n}$ is represented by a function decomposed with lower order interactions:

$$
f(x) \equiv f_{0}+\sum_{i} f_{i}\left(x_{i}\right)+\sum_{i<j} f_{i j}\left(x_{i}, x_{j}\right)+\cdots+f_{1,2, \cdots, n}\left(x_{1}, x_{2}, \ldots, x_{n}\right) .
$$

This expansion is exact and exists for any integrable and measurable function $f$, but it is not unique. There is a rich variety of such expansions depending on the projection operator used to construct them. The most used in statistics is the ANOVA-HDMR where the low dimensional functions are defined by

$$
\begin{gathered}
f_{0}^{A}(x) \equiv P_{0}^{A} f(x)=\int_{\mathbf{D}} f(x) d \mu(x), \\
f_{i}^{A}\left(x_{i}\right) \equiv P_{i}^{A} f(x)=\int_{\mathbf{D}_{i}} f(x) \prod_{i \neq j} d \mu_{j}\left(x_{j}\right)-P_{0}^{A} f(x), \\
f_{i_{1} \ldots i_{l}}^{A}\left(x_{i_{1}}, \ldots, x_{x_{l}}\right) \equiv P_{i_{1} \ldots i_{l}}^{A} f(x)=\int_{\mathbf{D}_{i_{1} \ldots i_{l}}} f(x) \prod_{k \in\left\{\left\{_{1} \ldots i_{l}\right\}\right.} d \mu_{k}\left(x_{k}\right)-\sum_{j_{1}<\ldots<j_{l-1} \in\left\{i_{1} \ldots i_{l}\right\}} P_{j_{1} \ldots j_{l-1}}^{A} f(x) \\
-\ldots-\sum_{j \in\left\{i_{1} \ldots i_{l}\right\}} P_{j}^{A} f(x)-P_{0}^{A} f(x),
\end{gathered}
$$

where $\mathbf{D}_{i_{1} \ldots i_{l}} \subset \mathbf{D}$ is the hypercube excluding indices $i_{1}, \ldots, i_{l}$ and $\mu$ is the product measure $\mu(x)=\prod_{i} \mu_{i}\left(x_{i}\right)$. This expansion can be used to express the total variance of $f$, by noting that

$$
\begin{gathered}
D \equiv \mathbf{E}\left[f-f_{0}\right]^{2}=\sum_{i} D_{i}+\sum_{i<j} D_{i j}+\cdots+D_{1,2, \ldots, n}, \\
D_{i_{1} \ldots i_{l}}=\int_{\mathbf{D}^{i_{1} \ldots i_{l}}}\left(f_{i_{1} \ldots i_{l}}^{A}\right)^{2} \prod_{k \in\left\{i_{1} \ldots i_{1}\right\}} d \mu_{k}\left(x_{k}\right) .
\end{gathered}
$$

However, the high-dimensional integrals in the ANOVA-HDMR expansion are computationally expensive to evaluate.

An alternative expansion is the cut-HDMR, that is built by superposition of hyperplanes passing through the cut center $y=\left(y_{1}, \ldots, y_{n}\right)$ :

$$
\begin{gathered}
f_{0}^{C}(x) \equiv P_{0}^{C} f(x)=f(y), \\
f_{i}^{C}\left(x_{i}\right) \equiv P_{i}^{C} f(x)=f^{i}\left(x_{i}\right)-P_{0}^{C} f(x), \\
f_{i_{1} \ldots i_{l}}^{C}\left(x_{i_{1}}, \ldots, x_{x_{l}}\right) \equiv P_{i_{1} \ldots i_{l}}^{C} f(x)=f^{i_{1}, \ldots, i_{l}}\left(x_{i_{1}}, \ldots, x_{x_{l}}\right)-\sum_{j_{1}<\ldots<j_{l-1} \in\left\{i_{1} \ldots i_{l}\right\}} P_{j_{1} \ldots j_{l-1}}^{C} f(x) \\
-\ldots-\sum_{j \in\left\{i_{1} \ldots i_{l}\right\}} P_{j}^{C} f(x)-P_{0}^{C} f(x),
\end{gathered}
$$

where $f^{i_{1}, \ldots, i_{l}}\left(x_{i_{1}}, \ldots, x_{x_{l}}\right)$ is the function $f(x)$ with all the remaining variables set to $y$. This expansion requires the evaluation of the function $f$ on lines, planes and hyperplanes passing through the cut center.

If cut-HDMR is a good approximation of $f$ at order $L$, i.e. considering up to $L$-terms interactions in (7), such expansion can be used for the computation of ANOVA-HDMR in place of the original function. This reduces the computational cost dramatically: let $n$ be the number of parameters and $S$ the number of samples taken along each direction (being them MC samples or cubature points), then the cost of constructing cut-HDMR is

$$
\sum_{i=0}^{L} \frac{n !}{(n-i) ! i !}(s-1)^{i}
$$

\subsection{Total Sensitivity Index}

The main task of Sensitivity Analysis is to quantify the sensitivity of the output with respect to the input. In particular it's important to know how much of this sensitivity is accountable to a particular parameter. With the focus on global sensitivity analysis, the sensitivity of the system to a particular parameter can be expressed by the variance of the output associated to that particular input.

One approach to this question is to consider each parameter separately and to apply one of the UQ techniques introduced in section 3.1. This approach goes by the name of one-at-a-time analysis. This technique is useful to get a first overview of the system. However, this technique lacks an analysis of the interaction between input 


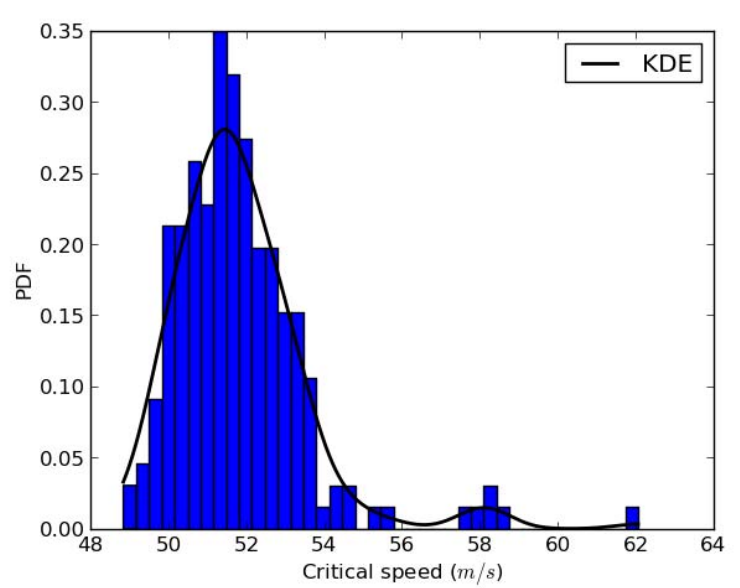

Fig. 3: Histogram of the Critical Speed obtained using Fig. 4: Pie plot of the Total Sensitivity Indices on the Latin Hypercube sampling and the estimated density reduced stochastic model, where only the most function (KDE) obtained using Kernel Smoothing.

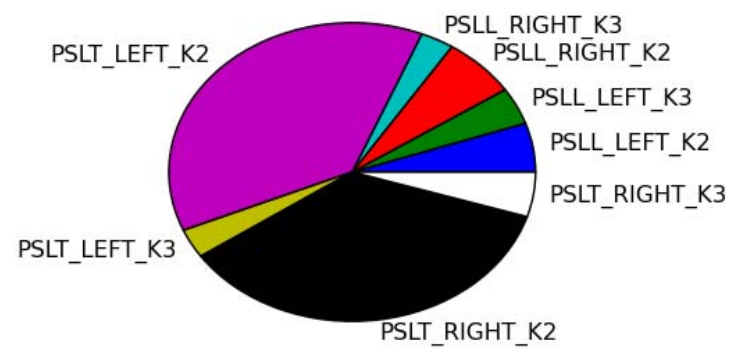

influential components are analyzed. (See Table 1 for an explanation of the notation used)

parameters, which in many cases is important.

A better analysis can be achieved using the method of Sobol' [14]. Here single sensitivity measures are given by

$$
S_{i_{1}, \ldots, i_{l}}=\frac{D_{i_{1}, \ldots, i_{l}}}{D} \quad \text { for } 1 \leq i_{1}<\cdots<i_{l} \leq n,
$$

where $D$ and $D_{i_{1}, \ldots, i_{l}}$ are defined according to (9). These express the amount of total variance that is accountable to a particular combination $i_{1}, \ldots, i_{l}$ of parameters. The Total Sensitivity Index (TSI) is the total contribution of a particular parameter to the total variance, including interactions with other parameters. It can be expressed by

$$
T S(i)=1-S_{\neg i},
$$

where $S_{\neg i}$ is the sum of all $S_{i_{1}, \ldots, i_{l}}$ that does not involve parameter $i$.

These total sensitivity indices can be approximated using sampling based methods in order to evaluate the integrals involved in (9). Alternatively, [4] suggests to use cut-HDMR and cubature rules in the following manner:

1. Compute the cut-HDMR expansion on cubature nodes for the input distributions.

2. Derive the approximated ANOVA-HDMR expansion from the cut-HDMR.

3. Compute the Total Sensitivity Indices from the ANOVA-HDMR.

This approach gives the freedom of selecting the level of accuracy for the HDMR expansion depending on the level of interaction between parameters. The truncation order $L$ of the ANOVA-HDMR can be selected and the accuracy of such expansion can be assessed using the concept of "effective dimension" of the system: for $q \leq 1$, the effective dimension of the integrand $f$ is an integer $L$ such that

$$
\sum_{0<t \mid \leq L} D_{t} \geq q D \text {, }
$$

where $t$ is a multi-index $i_{1}, \ldots, i_{l}$ and $|t|$ is the cardinality of such multi-index. The parameter $q$ is chosen based on a compromise between accuracy and computational cost.

\section{SENSITIVITY ANALYSIS ON RAILWAY VEHICLE DYNAMICS}

The study of uncertainty propagation and sensitivity analysis through dynamical systems is a computationally expensive task. In this analysis we adopt a collocation approach, where we study the behaviors of ensembles of realizations. From the algorithmic point of view, the quality of a method is measured in the number of realizations needed in order to infer the same statistics. Each realization is the result of an Initial Value Problem (IVP) computed using the program DYnamics Train SImulation (DYTSI) developed in [5], where the model presented in section 2 has been set up and the IVP has been solved using the Explicit Runge-Kutta-Fehlberg method ERKF34 [15]. An explicit solver has been used in light of the analysis performed in [16], where it was found that the hunting motion could be missed by implicit solvers, used with relaxed tolerances, due to numerical damping. In particular implicit solvers are frequently used for stiff problems, like the one treated here, because their step-size is bounded 
by accuracy constraints instead of stability. However, the detection of hunting motion requires the selection of strict tolerances, reducing the allowable step-sizes and making the implicit methods more expensive than the explicit ones. Since the collocation approach for UQ involves the computation of completely independent realizations, this allows for a straightforward parallelization of the computations on clusters. Thus, 25 nodes of the DTU cluster have been used to speed up the following analysis.

The first step in the analysis of a stochastic system is the characterization of the probability distribution of the QoI. Since the complete model has co-dimension 24, a traditional sampling method, among the ones presented in section 3.1, is the most suited for the task of approximating the integrals in eq. (4). Fig. 3 shows the histogram of the computed critical speeds with respect to the uncertainty in the suspension components. In order to speed up the convergence, we used 200 samples generated with the Latin Hyper Cube method [17]. Kernel smoothing [10] has been used to estimate the density function according to this histogram. The estimated mean and variance are $\bar{\mu}_{v}=51.83 \mathrm{~m} / \mathrm{s}$ and $\bar{\sigma}_{v}=4.07 \mathrm{~m}^{2} / \mathrm{s}^{2}$.

\begin{tabular}{|c|c|c|c|c|c|}
\hline \multirow{2}{*}{ Suspension } & \multirow{2}{*}{$\begin{array}{c}\text { One-at-a-time } \\
\bar{\sigma}_{v}\end{array}$} & \multicolumn{2}{|c|}{ ANOVA } & \multicolumn{2}{|c|}{ ANOVA - Refined } \\
\hline & & $\bar{\sigma}_{v}$ & Tot. Sensitivity & $\bar{\sigma}_{v}$ & Tot. Sensitivity \\
\hline PSLL_LEFT_K1 & 0.00 & 0.03 & 0.01 & & \\
\hline PSLL_LEFT_K2 & 0.06 & 0.18 & 0.06 & 0.18 & 0.09 \\
\hline PSLL_LEFT_K3 & 0.02 & 0.13 & 0.04 & 0.14 & 0.07 \\
\hline PSLL_RIGHT_K1 & 0.00 & 0.05 & 0.02 & & \\
\hline PSLL_RIGHT_K2 & 0.06 & 0.17 & 0.06 & 0.22 & 0.11 \\
\hline PSLL_RIGHT_K3 & 0.03 & 0.17 & 0.06 & 0.10 & 0.05 \\
\hline PSLT_LEFT_K1 & 0.00 & 0.02 & 0.01 & & \\
\hline PSLT_LEFT_K2 & 0.54 & 1.71 & 0.56 & 1.29 & 0.63 \\
\hline PSLT_LEFT_K3 & 0.14 & 0.20 & 0.07 & 0.11 & 0.05 \\
\hline PSLT_RIGHT_K1 & 0.00 & 0.05 & 0.02 & & \\
\hline PSLT_RIGHT_K2 & 0.55 & 1.73 & 0.56 & 1.22 & 0.59 \\
\hline PSLT_RIGHT_K3 & 0.03 & 0.13 & 0.04 & 0.17 & 0.08 \\
\hline SSL_LEFT_K4 & 0.00 & 0.01 & 0.00 & & \\
\hline SSL_LEFT_K5 & 0.00 & 0.01 & 0.00 & & \\
\hline SSL_LEFT_K6 & 0.00 & 0.02 & 0.01 & & \\
\hline SSL_LEFT_D1 & 0.00 & 0.02 & 0.01 & & \\
\hline SSL_LEFT_D2 & 0.02 & 0.04 & 0.01 & & \\
\hline SSL_LEFT_D6 & 0.00 & 0.02 & 0.01 & & \\
\hline SSL_RIGHT_K4 & 0.00 & 0.01 & 0.00 & & \\
\hline SSL_RIGHT_K5 & 0.00 & 0.00 & 0.00 & & \\
\hline SSL_RIGHT_K6 & 0.00 & 0.02 & 0.01 & & \\
\hline SSL_RIGHT_D1 & 0.00 & 0.03 & 0.01 & & \\
\hline SSL_RIGHT_D2 & 0.00 & 0.04 & 0.01 & & \\
\hline SSL_RIGHT_D3 & 0.00 & 0.02 & 0.01 & & \\
\hline
\end{tabular}

Table 1: Variances and Total Sensitivity Indices obtained using the One-at-a-time analysis, the ANOVA expansion of the complete model and the more accurate ANOVA expansion of the reduced model. The naming convention used for the suspensions works as follows. PSL and SSL stand for primary and secondary suspension of the leading bogie respectively. The following $L$ and $T$ in the primary suspension stand for leading and trailing wheel sets. The last part of the nomenclature refers to the particular suspension components as shown in Fig. 1.

\subsection{One-at-a-time analysis}

When each suspension component is considered independently from the others, the estimation problem in (4) is reduced to the calculation of a 1-dimensional integral. This task can be readily achieved by quadrature rules that have proven to be computationally more efficient on problems of this dimensionality than sampling methods [3]. Fourth order quadrature rules have been used to approximate the variances due to the single components. The convergence of this method enables a check of accuracy through the decay of the expansion coefficients of the target function [3].

The second column in Table 1 lists the results of such analysis. The amount of variance described by this analysis is given by the sum of all the variances: $\bar{\sigma}_{\mathrm{OAT}}=1.47 \mathrm{~m}^{2} / \mathrm{s}^{2}$. This quantity is far from representing the total variance of the stochastic system, suggesting that interactions between suspension components are important. Anyway the method is useful to make a first guess about which components are the most important: the critical speed of the railway vehicle model analyzed in this work shows a strong sensitivity related to the longitudinal 
springs (K2) in the trailing wheel set.

\subsection{Total Sensitivity Analysis}

The technique outlined in section 3.3 can fulfill three important tasks: taking into account parameter interactions, performing the analysis with a limited number of realizations and enabling an error control in the approximation. In a first stage we consider the full stochastic model and we construct a cut-HDMR expansion which takes into account $2^{\text {nd }}$ order interactions and describes the target function through $2^{\text {nd }}$ order polynomials, computing the realizations for up to 2-dimensional cubature rules. The ANOVA-HDMR expansion of the cut-HDMR expansion can be quickly computed, due to the low dimensionality of the single terms in (10). At this point, the $D_{i_{1}, \ldots, i_{l}}$ values in (9) can be obtained and the "effective dimensionality" of the target function, given by (14) for $q=0.95$, is found to be $L=2$. This confirms that the $1^{\text {st }}$ and $2^{\text {nd }}$ order interactions are sufficient to describe most of the variance. The third and fourth column of Table 1 list the total variances induced by each parameter, including interactions with other parameters, and the Sobol' total sensitivity indices.

Once the first approximation of the sensitivities is obtained, the parameters with the lowest sensitivity indices can be fixed to their nominal values and we can perform a more accurate analysis of the remaining stochastic system. Longitudinal and vertical springs (K2 and K3) in the primary suspensions have shown to be very influential for the critical speed of the analyzed model, thus a new cut-HDMR expansion, with $2^{\text {nd }}$ order interactions and $4^{\text {th }}$ order polynomial approximation is constructed. The resulting total variances and total sensitivity indices are listed in the fifth and sixth column of Table 1. A visual representation of the sensitivity indices is shown in the pie chart in Fig. 4.

The results obtained by the one-at-a-time analysis are confirmed here by the total sensitivity analysis, but we stress that the latter provide a higher reliability because they describe a bigger part of the total variance of the complete stochastic system.

\subsection{Discussion of the obtained results}

Even if the results obtained are formally correct, the interpretation of such results can raise some questions. A railway engineer might wonder why the yaw dampers D2 are not listed among the most important by the sensitivity analysis. The yaw dampers in the secondary suspension are known to provide stability to the vehicle ride, helping to increase its critical speed. This result is true also with the vehicle model considered here, in fact low values of D2 cause a drastic worsening of the ride stability. However, the total sensitivity indices embed the probability distributions of the uncertain parameters in the global sensitivity analysis: the impact of a component is weighted according to these distributions. Thus we say that the yaw damper has little influence on the riding stability with respect to the distributions chosen. A change in the distributions can dramatically change these results, thus particular care should be taken with the quantification of the source of uncertainty.

Finally, observe that, even if they are not as important as the primary suspension components, the yaw dampers seem to be the most important components among the secondary suspensions.

\subsection{Remarks on sensitivity analysis on non-linear dynamics}

Uncertainty quantification and sensitivity analysis require a rigorous preliminary formulation of the stochastic system, its sources of uncertainty and the Quantities of Interest. We already mentioned in section 2.2 that in this work the characterization of the sources of uncertainty was bypassed by assuming Gaussian distributions for all the parameters, without loss of generality for the methods presented. The selection of the QoI, however, merits some more discussion. In section 2.1 the continuation method used to estimate the critical speed was presented and the threshold used to determine the end of the hunting motion was chosen in a conservative way, as it is shown in Fig. 2b. However, the value of the computed critical speed will depend also on the deceleration chosen for the continuation method, i.e. the computed critical speed will be exact in the limit when the deceleration goes to zero. Of course, the exact computation of the critical speed is not computationally feasible. With the limited computational resources available, we then chose a fixed deceleration coefficient for the continuation method, and thus we introduced numerical uncertainty in the computations. Therefore, the variance expressed from the analysis is given both by the variance due to the stochastic system and the variance introduced by the computation of the QoI. This is, however, a conservative consequence, meaning that a decision taken on the basis of the computed results is at least as safe as a decision taken using the "exact results".

\section{CONCLUSIONS}

Sensitivity analysis is of critical importance on a wide range of engineering applications. The traditional approach of local sensitivity analysis is useful in order to characterize the behavior of a dynamical system in the vicinity of the nominal values of its parameters, but it fails in describing wider ranges of variations, e.g., caused by long-term 
wear. The global sensitivity analysis aims at representing these bigger variations and at the same time it embeds the probability distributions of the parameters in the analysis. This enables the engineer to take decisions based on the risk of a certain event to happen.

Wrongly approached, global sensitivity analysis can turn to be a computationally expensive or even prohibitive task. In this work a collection of techniques are used in order to accelerate such analysis for a high-co-dimensional problem. Each of the techniques used allows for a control of the accuracy, e.g., in terms of convergence rate for the cubature rules in section 3.1 and the "effective dimension" in section 3.3. This makes the framework flexible and easy to be adapted to problems with more diversified distributions and target functions.

The analysis performed on the half wagon equipped with a Cooperrider bogie shows a high importance of the longitudinal primary suspensions, and this reflects the connection between hunting and yaw motion. Furthermore, the importance of the yaw damper in the secondary suspensions is confirmed, even if its influence is little compared to the primary suspensions.

It is important to notice that the same settings for global sensitivity analysis can be used for the investigation of different Quantities of Interests, such as wear in curved tracks, angle of attack etc., once they have been properly defined. Furthermore, the "non-intrusive" approach taken allows the engineer to use closed software for the computations. The machinery for sensitivity analysis needs only to be wrapped around it, without additional implementation efforts.

\section{References}

[1] C. Funfschilling, G. Perrin and S. Kraft, "Propagation of variability in railway dynamic simulations: application to virtual homologation," Vehicle System Dynamics, vol. 50, no. sup1, pp. 245-261, 2012.

[2] L. Mazzola and S. Bruni, "Effect of Suspension Parameter Uncertainty on the Dynamic Behaviour of Railway Vehicles," Applied Mechanics and Materials , vol. 104, pp. 177-185, 2011.

[3] D. Bigoni, A. P. Engsig-Karup and H. True, "Comparison of Classical and Modern Uncertainty Quantification Methods for the Calculation of Critical Speeds in Railway Vehicle Dynamics," in 13th mini conference on vehicle system dynamics, identification and anomalies, Budapest, 2012.

[4] Z. Gao and J. S. Hesthaven, "Efficient solution of ordinary differential equations with high-dimensional parametrized uncertainty," Communications in Computational Physics, vol. 10, no. 2, pp. 253-286, 2011.

[5] D. Bigoni, "Curving Dynamics in High Speed Trains," Technical University of Denmark, DTU Informatics, Kgs. Lyngby, Denmark, 2011, MSc Thesis, http://www2.imm.dtu.dk/pubdb/views/publication_details.php?id=6105.

[6] W. Kik and D. Moelle, "ACRadSchiene - To create or Approximate Wheel/Rail profiles - Tutorial".

[7] J. J. Kalker, "Wheel-rail rolling contact theory," Wear, vol. 144, no. 1-2, pp. 243-261, 1991.

[8] Z. Y. Shen, J. K. Hedrick and J. A. Elkins, "A comparison of alternative creep-force models for rail vehicle dynamic analysis," in 8th IASVD Symposium, 1984.

[9] H. True, "Multiple attractors and critical parameters and how to find them numerically: the right, the wrong and the gambling way," Vehicle System Dynamics, pp. 1-17, 2012.

[10] T. Hastie, R. Tibishirani and J. Firedman, "Kernel Smoothing Methods," in The elements of statistical learning, 10th ed., 2013, pp. 191-218.

[11] W. Gautschi, Orthogonal Polynomials: Computation and Approximation, Oxford: Oxford University Press, 2004.

[12] K. Petras, "Smolyak cubature of given polynomial degree with few nodes for increasing dimension," Numerische Mathematik, vol. 93, no. 4, pp. 729-753 , 2003.

[13] H. Rabitz and F. A. Ömer, "Managing the Tyranny of Parameters in Mathematical Modelling of Physical Systems," in Sensitivity Analysis, Chichester, West Sussex: John Wiley \& Sons Ltd., 2000, pp. 199-223.

[14] K. Chan, S. Tarantola, A. Saltelli and I. M. Sobol', "Variance-Based Methods," in Sensitivity Analysis, Chichester, West Sussex: John Wiley \& Sons Ltd., 2000, pp. 168-197.

[15] E. Hairer, S. P. Nørsett and G. Wanner, Solving Ordinary Differential Equations I: Nonstiff problems, second revision ed., Berlin, Wien, New York: Springer Series in Computational Mathematics, Springer-Verlag, 1991.

[16] H. True, A. P. Engsig-Karup and D. Bigoni, "On the Numerical and Computational Aspects of Non-Smoothnesses that occur in Railway Vehicle Dynamics," Mathematics and Computers in Simulation, 2012.

[17] M. D. McKay, R. J. Beckman and W. J. Conover, "A comparison of three methods of selecting values of input variables in the analysis of output from a computer code.," Technometrics, vol. 21, pp. 239-245, 1979. 